\title{
The Ground State Energy per Particle for Infinite Particle Quantum Systems ${ }^{\star}$
}

\author{
D. Isaacson \\ Rutgers University, New Brunswick, New Jersey, USA
}

\begin{abstract}
We give a simple proof that the ground state energy per particle for several interacting particle systems is monotone and bounded as the number of particles increases. Some of the systems for which the proof holds are anharmonic oscillator approximations to $|\boldsymbol{\phi}|_{d}^{4}$ quantum fields, many body Schrödinger operators with nearest and next to nearest neighbor couplings, and systems whose energy is given by operators which are not restricted to being differential operators.
\end{abstract}

It is well known that if we pinch a vibrating system of particles, or clamp a vibrating membrane the fundamental frequencies increase. Similarly if we tear a vibrating membrane its fundamental frequency decreases. Some of the mathematical consequences of these observations, in classical physics, are that imposing Dirichlet or Neumann boundary conditions on $-\Delta$, (or finite difference approximations to $-\Delta$ ) along a curve in the interior of some bounded region, raises or lowers its eigenvalues [1].

Some of the mathematical consequences of these observations in quantum physics are described in this paper. We show in particular that the ground state energy per particle, for the sequence of coupled anharmonic oscillator Schrödinger operators with Dirichlet boundary conditions used in the "doubling algorithm" in [2] to numerically approximate the $\phi_{2}^{4}$ quantum field theory, is monotone decreasing.

We next show that the ground state energy per particle, for the sequence of coupled anharmonic oscillator Schrödinger operators with Neumann Boundary conditions used in [2] to approximate the $\phi_{2}^{4}$ theory, is monotone increasing.

We also show that the energy per particle is bounded from above and below in both of the preceding cases and hence it converges to a limit as the number of particles becomes infinite.

* Research partially supported by the National Science Foundation under grant No. MCS-77-03568 
We remark that our method of proof works for a larger class of coupled systems which includes spherically symmetric anharmonic oscillator approximations to $|\phi|_{d}^{4}$ quantum field theories. The method of proof also works for couplings between the oscillators which are not "nearest neighbors". In fact, as is easily seen, the proof will work for a much larger class of interacting systems where the energy operator for each individual particle need not even be a differential operator.

We illustrate the proof in the simplest case, the $n$-oscillator approximation (with Dirichlet boundary conditions) to the $\phi_{2}^{4}$ quantum field theory [2]. Let $H_{N}$ denote the $N$-oscillator Schrödinger operator

$$
H_{N} \equiv \sum_{j=1}^{N} A_{j}-\sum_{j=1}^{N-1} q_{j} q_{j+1},
$$

where for $a, b, c$, and $d$ real numbers with $a>0$

$$
A_{j} \equiv \frac{1}{2}\left[-\frac{\partial^{2}}{\partial q_{j}^{2}}+a q_{j}^{4}+b q_{j}^{2}+c q_{j}+d\right] \text {. }
$$

The operators $H_{N}$ have a complete orthonormal set of eigenfunctions

$$
\Omega_{j}^{(N)}=\Omega_{j}^{(N)}\left(q_{1}, q_{2}, \ldots, q_{N}\right)
$$

whose eigenvalues we denote by $E_{j}(N)$, where

$$
E_{0}(N)<E_{1}(N) \leqq E_{2}(N) \leqq \ldots
$$

Theorem I. If $E_{0}(N)$ denotes the lowest eigenvalue of the $N$-oscillator "Dirichlet" operator (1) then,

(i) $p(n) \equiv E_{0}\left(2^{n}\right) / 2^{n}$, is monotone decreasing as $n$ increases.

(ii) $p(n)$ is bounded above and below for all $n$.

(iii) The limit as $n \rightarrow \infty$ of $p(n)$ exists.

The proof of (i) would be trivial if the system was not interacting, i.e. if the "coupling" term $\Sigma q_{j} q_{j+1}$ was not present in (1) because then $E_{0}(N)=N E_{0}(1)$.

In the interacting or coupled case we use a variational calculation to prove monotonicity by showing that "pinching the system in the middle" raises its energy. We have that

$$
\begin{aligned}
E_{0}(2 N) & =\inf _{\psi \in L_{2}\left(R^{2 N}\right)}\left\langle\psi, H_{2 N} \psi\right\rangle \\
& \leqq\left\langle\Omega, H_{2 N} \Omega\right\rangle
\end{aligned}
$$

where we take

$$
\Omega\left(q_{1}, \ldots, q_{N}, q_{N+1}, \ldots, q_{2 N}\right)=\Omega_{0}^{(N)}\left(q_{1} \ldots q_{N}\right) \Omega_{0}^{(N)}\left(q_{N+1} \ldots q_{2 N}\right) .
$$

Observe that $H_{2 N}$ may be written as

$$
H_{2 N}=H_{N}^{L}+H_{N}^{R}-q_{N} q_{N+1}
$$

where

$$
H_{N}^{L} \equiv \sum_{j=1}^{N} A_{j}-\sum_{j=1}^{N-1} q_{j} q_{j+1}
$$


and

$$
H_{N}^{R}=\sum_{j=N+1}^{2 N} A_{j}-\sum_{j=N+1}^{2 N-1} q_{j} q_{j+1} .
$$

Thus from (5) and (6)

$$
E_{0}(2 N) \leqq 2 E_{0}(N)-Q_{N} Q_{N+1},
$$

where

$$
\begin{aligned}
Q_{N} & \equiv\left\langle\Omega_{0}^{(N)}\left(q_{1} \ldots q_{N}\right), q_{N} \Omega_{0}^{(N)}\left(q_{1} \ldots q_{N}\right)\right\rangle \\
Q_{N+1} & \equiv\left\langle\Omega_{0}^{(N)}\left(q_{N+1} \ldots q_{2 N}\right), q_{N+1} \Omega_{0}^{(N)}\left(q_{N+1} \ldots q_{2 N}\right)\right\rangle .
\end{aligned}
$$

We now use the facts that $E_{0}^{(N)}$ is non-degenerate, and $H_{N}$ commutes with the unitary map $R$ given by

$$
R \psi\left(q_{1} \ldots q_{N}\right)=\psi\left(q_{N} \ldots q_{1}\right)
$$

to conclude that $Q_{N}=Q_{N+1}$.

Therefore

$$
E_{0}(2 N) \leqq 2 E_{0}(N)-Q_{N}^{2} \leqq 2 E_{0}(N) \text {. }
$$

This inequality illustrates the fact that the "pinched" system [whose ground state energy is $\left.2 E_{0}(N)\right]$ has higher energy than the original system. It follows from (13) that

$$
\frac{E_{0}(2 N)}{2 N} \leqq \frac{E_{0}(N)}{N}
$$

and therefore the function $p(N)=E_{0}\left(2^{N}\right) / 2^{N}$ is monotone decreasing as $N$ increases.

We remark that the proof just given would hold if the $q_{j}$ were replaced by vectors

$$
\begin{aligned}
& \mathbf{q}_{j}=\left(q_{j}^{1}, q_{j}^{2}, \ldots, q_{j}^{m}\right) \text { and } \\
& \frac{\partial^{2}}{\partial q_{j}^{2}} \text { replaced by Laplacians } \sum_{k=1}^{m}\left(\partial / \partial q_{j}^{k}\right)^{2}
\end{aligned}
$$

(the $H_{N}$ 's would then be approximations to the $|\phi|_{2}^{4}$ theory). It is also clear that the proof would work if we took $A_{j}=-\partial^{2} / \partial q_{j}^{2}+V\left(q_{j}\right)$ for a large class of functions $V$. In fact the $A_{j}$ need not even be differential operators for the above proof to work.

We now prove that $p(n)$ is a bounded function of $n$ by showing that $E_{0}(N) / N$ is bounded. As quadratic forms we have that

$$
\frac{-q_{j}^{2}-q_{j+1}^{2}}{2} \leqq-q_{j} q_{j+1} \leqq \frac{q_{j}^{2}+q_{j+1}^{2}}{2}
$$

and hence

$$
\begin{aligned}
& \sum_{j=2}^{N-1} A_{j}-q_{j}^{2}+A_{1}-\frac{q_{1}^{2}}{2}+A_{N}-\frac{q_{N}^{2}}{2} \\
& \quad \leqq H_{N} \leqq \sum_{j=2}^{N-1} A_{j}+q_{j}^{2}+A_{1}+\frac{q_{1}^{2}}{2}+A_{N}+\frac{q_{N}^{2}}{2}
\end{aligned}
$$


which implies that

$$
\sum_{j=1}^{N} A_{j}-q_{j}^{2} \leqq H_{N} \leqq \sum_{j=1}^{N} A_{j}+q_{j}^{2}
$$

If we denote the lowest eigenvalues of $A_{j} \pm q_{j}^{2}$ by $E_{0}^{ \pm}(1)$ then we have that

$$
N E_{0}^{-}(1) \leqq E_{0}(N) \leqq N E_{0}^{+}(1)
$$

from which it follows that

$$
E_{0}^{-}(1) \leqq \frac{E_{0}(N)}{N} \leqq E_{0}^{+}(1)
$$

proving the boundedness of $p(n)$. Part (iii) follows from (i) and (ii).

If instead of the "Dirichlet operators" we had used "Neumann operators" [2]

$$
K_{N} \equiv \sum_{j=2}^{N-1} A_{j}+\left(\frac{A_{1}-q_{1}^{2}}{2}\right)+\left(\frac{A_{N}-q_{N}^{2}}{2}\right)-\sum_{j=1}^{N-1} q_{j} q_{j+1} .
$$

We have that

$$
K_{2 N}=K_{N}^{L}+K_{N}^{R}+\left(\frac{q_{N+1}-q_{N}}{2}\right)^{2},
$$

where

$$
\begin{aligned}
K_{N}^{L} & =\sum_{j=2}^{N-1} A_{j}+\left(\frac{A_{1}-q_{1}^{2}}{2}\right)+\left(\frac{A_{N}-q_{N}^{2}}{2}\right)-\sum_{j=1}^{N-1} q_{j} q_{j+1} \\
K_{N}^{R} & =\sum_{j=N+1}^{2 N-1} A_{j}+\left(\frac{A_{N+1}-q_{N+1}^{2}}{2}\right)+\left(\frac{A_{2 N}-q_{2 N}^{2}}{2}\right)-\sum_{j=N+1}^{2 N-1} q_{j} q_{j+1} .
\end{aligned}
$$

Hence as quadratic forms

$$
K_{2 N} \geqq K_{N}^{L}+K_{N}^{R}
$$

which implies that the lowest eigenvalue $E_{0}^{K}(2 N)$ of $K_{2 N}$ is larger than the lowest eigenvalue $2 E_{0}^{K}(N)$ of the system "torn in the middle". Thus

$$
\frac{E_{0}^{K}(2 N)}{2 N} \geqq \frac{E_{0}^{K}(N)}{N}
$$

which implies that $p^{K}(n) \equiv E_{0}^{K}\left(2^{n}\right) / 2^{n}$ is an increasing function of $n$. The fact that $p^{K}(n)$ is bounded is proved as in the "Dirichlet" case. We have therefore proven

Theorem II. If $E_{0}^{K}(N)$ denotes the lowest eigenvalue of the $N$-oscillator Neumann operator $K_{N}(20)$ then

(i) $p^{K}(n)=E_{0}\left(2^{n}\right) / 2^{n}$ is monotone increasing as $n$ increases.

(ii) $p^{K}(n)$ is bounded in $n$.

(iii) The limit as $n \rightarrow \infty$ of $p^{K}(n)$ exists.

To illustrate the method of proof when the system has more than nearest neighbor interactions, we prove monotonicity of the ground state energy per particle for a system of oscillators with nearest and next nearest neighbor coupling 
whose energy operator is

$$
G_{N}=\sum_{j=1}^{N} B_{j}+\sum_{j=1}^{N-2} q_{j} q_{j+2}-\sum_{j=1}^{N-1} q_{j} q_{j+1},
$$

where, for example, we could take, for $a>0$,

$$
B_{j} \equiv-\frac{\partial^{2}}{\partial q_{j}^{2}}+a q_{j}^{6}+b q_{j}^{4}+c q_{j}^{2}+\cos \left(q_{j}\right)+d .
$$

Denote the lowest eigenvalue of $G_{N}$ by $E_{0}^{G}(N)$ and its eigenfunction by $\Phi_{0}^{(N)}\left(q_{1} \ldots q_{N}\right)$. As in the proof of Theorem I we have that

$$
E_{0}^{G}(2 N) \leqq\left\langle\Omega, G_{2 N} \Omega\right\rangle,
$$

where

$$
\Omega=\Phi_{0}^{(N)}\left(q_{1} \ldots q_{N}\right) \Phi_{0}^{(N)}\left(q_{N+1} \ldots q_{2 N}\right)
$$

and

$$
G_{2 N}=G_{N}^{L}+G_{N}^{R}-q_{N} q_{N+1}+q_{N-1} q_{N+1}+q_{N} q_{N+2},
$$

where

$$
\begin{aligned}
G_{N}^{L} & =\sum_{j=1}^{N} B_{j}-\sum_{j=1}^{N-1} q_{j} q_{j+1}+\sum_{j=1}^{N-2} q_{j} q_{j+2} \\
G_{N}^{R} & =\sum_{j=N+1}^{2 N} B_{j}-\sum_{j=N+1}^{2 N-1} q_{j} q_{j+1}+\sum_{j=N+1}^{2 N-2} q_{j} q_{j+2} .
\end{aligned}
$$

From (27) and (29) it follows that

$$
E_{0}^{G}(2 N) \leqq 2 E_{0}^{G}(N)-Q_{N} Q_{N+1}+Q_{N-1} Q_{N+1}+Q_{N} Q_{N+2},
$$

where

$$
\begin{aligned}
Q_{N} & =\left\langle\Phi_{0}^{(N)}\left(q_{1} \ldots q_{N}\right), q_{N} \Phi_{0}^{(N)}\left(q_{1} \ldots q_{N}\right)\right\rangle \\
Q_{N+1} & =\left\langle\Phi_{0}^{(N)}\left(q_{N+1} \ldots q_{2 N}\right), q_{N+1} \Phi_{0}^{(N)}\left(q_{N+1} \ldots q_{2 N}\right)\right\rangle \\
Q_{N+2} & =\left\langle\Phi_{0}^{(N)}\left(q_{N+1} \ldots q_{2 N}\right), q_{N+2} \Phi_{0}^{(N)}\left(q_{N+1} \ldots q_{2 N}\right)\right\rangle .
\end{aligned}
$$

However $Q_{N}=Q_{N+1}=Q_{N+2}=0$ here because $G_{N}$ commutes with the unitary operator $S$ which acts by

$$
S \psi\left(q_{1} \ldots q_{N}\right) \equiv \psi\left(-q_{1} \ldots-q_{N}\right) .
$$

We have therefore shown that $p^{G}(n) \equiv E_{0}^{G}\left(2^{n}\right) / 2^{n}$ is monotone decreasing as $n$ increases.

The last system that we illustrate this method of proving monotonicity of the energy per particle is a Dirichlet anharmonic oscillator approximation to the $\phi_{d}^{4}$ field theory, were for simplicity we choose $d=3$.

Let $H(M, N)$ be given by

$$
\begin{aligned}
H(M, N) \equiv & \sum_{j=1}^{M} \sum_{k=1}^{N} A_{j, k}-\sum_{j=1}^{M-1} \sum_{k=1}^{N} q_{j, k} q_{j+1, k} \\
& -\sum_{j=1}^{M} \sum_{k=1}^{N-1} q_{j, k} q_{j, k+1},
\end{aligned}
$$


where for $a>0$

$$
A_{j, k} \equiv \frac{1}{2}\left[-\frac{\partial^{2}}{\partial q_{j, k}^{2}}+a q_{j, k}^{4}+b q_{j, k}^{2}+c q_{j, k}+d\right] .
$$

If we let $E_{0}(M, N)$ denote the lowest eigenvalue of $H(M, N)$ then we have

\section{Theorem III}

(i) $p(n) \equiv E_{0}\left(2^{n}, 2^{n}\right) / 2^{2 n}$ is monotone decreasing as $n$ increases.

(ii) $p(n)$ is bounded.

(iii) The limit as $n \rightarrow \infty$ of $p(n)$ exists.

We prove (i) by showing that

$$
E_{0}(2 N, 2 N) \leqq 2 E_{0}(N, 2 N)
$$

and

$$
E_{0}(N, 2 N) \leqq 2 E_{0}(N, N)
$$

thus

$$
E_{0}(2 N, 2 N) \leqq 4 E_{0}(N, N)
$$

which yields (i).

We prove (36) by comparing the ground state energy of $H(2 N, 2 N)$ to that of a system "pinched down the middle" i.e. we write

$$
\begin{aligned}
H(2 N, 2 N)= & H^{L}(N, 2 N)+H^{R}(N, 2 N)-\sum_{k=1}^{2 N} q_{N, k} q_{N+1, k}, \\
H^{L}(N, 2 N)= & \sum_{j=1}^{N} \sum_{k=1}^{2 N} A_{j, k}-\sum_{j=1}^{N-1} \sum_{k=1}^{2 N} q_{j, k} q_{j+1, k}-\sum_{j=1}^{N} \sum_{k=1}^{2 N-1} q_{j, k} q_{j, k+1} \\
H^{R}(N, 2 N)= & \sum_{j=N+1}^{2 N} \sum_{k=1}^{2 N} A_{j, k}-\sum_{j=N+1}^{2 N-1} \sum_{k=1}^{2 N} q_{j, k} q_{j+1, k} \\
& -\sum_{j=N+1}^{2 N} \sum_{k=1}^{2 N-1} q_{j, k} q_{j, k+1} .
\end{aligned}
$$

As in the proof of Theorem I we have that

$$
E_{0}(2 N, 2 N) \leqq 2 E_{0}(N, 2 N) \text {. }
$$

The inequality (37) is proven similarly by "pinching" $H^{L}(N, 2 N)$ and $H^{R}(N, 2 N)$ across their middles.

\section{References}

1. Courant, R., Hilbert, D.: Methods of mathematical physics, Vol. 1. London: Wiley 1953

2. Isaacson, D., Marchesin, D., Paes-Leme, P.J.: Numerical methods for studying anharmonic oscillator approximations to the $\phi_{2}^{4}$ quantum field theory. Rutgers University Preprint, 1979

Communicated by A. Jaffe

Received October 21, 1979 